\title{
Le VIH au Canada - Rapport de surveillance, 2016
}

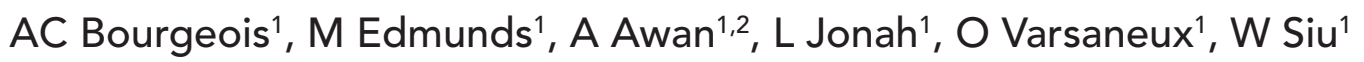

\section{Résumé}

Contexte : Le virus de l'immunodéficience humaine $(\mathrm{VIH})$ continue d'être un problème de santé publique, avec 2,1 millions de personnes nouvellement infectées en 2015. Bien que de nombreux pays à revenu élevé aient noté la diminution des taux de $\mathrm{VIH}$, les taux du Canada se sont stabilisés à 5,8 pour 100000 habitants entre 2013 et 2015.

Objectif : Fournir un aperçu descriptif des cas déclarés d'infection au VIH au Canada jusqu'en 2016 par provinces et territoires, sexe, âge, catégorie d'exposition et race/origine ethnique, l'accent étant mis sur les données les plus récentes.

Méthodologie : L'Agence de la santé publique du Canada (ASPC) surveille le VIH à travers le système national de surveillance du VIH/sida, Immigration, Réfugiés et Citoyenneté Canada, et le Programme de surveillance périnatale du VIH au Canada (PSPVC). Le système national de surveillance du VIH/sida est un système de surveillance passive basé sur les cas qui recueille des données non nominatives transmises volontairement par l'ensemble des provinces et territoires. Des données ont également été reçues d'Immigration, de Réfugiés et Citoyenneté Canada, et du PSPVC. Les données ont été recueillies, les tableaux et les figures ont été préparés, puis des statistiques descriptives ont été appliquées par l'ASPC, pour ensuite être validées par chaque province et territoire.

Résultats : Un total de 2344 nouveaux cas d'infection au VIH ont été diagnostiqués en 2016 au Canada, avec un total cumulatif de 84409 cas depuis 1985. Le taux de diagnostic national est passé de 5,8 pour 100000 habitants en 2015 à 6,4 pour 100000 habitants en 2016. La Saskatchewan a enregistré le plus haut taux de diagnostic provincial en 2016 (15,1 par 100000 habitants). En 2016, 76,6 \% des cas de VIH déclarés l'ont été parmi les hommes. Les adultes de 30 à 39 ans représentaient $28,7 \%$ de tous les cas déclarés. On a constaté la présence d'une répartition similaire par âge des cas de $\mathrm{VIH}$ entre les sexes, avec une augmentation notable de la proportion des personnes de 50 ans et plus au cours des cinq dernières années. La catégorie d'exposition des «hommes ayant des relations sexuelles avec d'autres hommes» a continué à représenter le plus grand nombre et la plus grande proportion de tous les cas déclarés d'infection au VIH chez les adultes (44,1\%). Quant aux catégories liées à la race ou à l'origine ethnique, les Caucasiens (40,4\%), les Afro-Américains (21,9\%) et les Autochtones $(21,2 \%)$ représentaient la plus grande proportion de cas.

Conclusion : En 2016, le Canada a vu une légère augmentation du nombre et du taux de cas d'infection au VIH déclarés en comparaison aux années précédentes. Bien que le taux de diagnostic soit inférieur à celui de toutes les années antérieures à 2012, il demeure le plus élevé des cinq dernières années. Comme un certain nombre de possibilités existent pour expliquer cette augmentation, une investigation et des données supplémentaires sont nécessaires afin de déterminer la cause et son importance.

\begin{abstract}
Affiliations
${ }^{1}$ Centre de la lutte contre les maladies transmissibles et les infections, Agence de la santé publique du Canada, Ottawa (Ontario)

2 Dalla Lana School of Public Health, University of Toronto, Toronto (Ontario)
\end{abstract}

Correspondance : HASS@ phac-aspc.gc.ca

Citation proposée : Bourgeois AC, Edmunds M, Awan A, Jonah L, Varsaneux O, Siu W. Le VIH au Canada - Rapport de surveillance, 2016 Relevé des maladies transmissibles au Canada. 2017;43(12):282-91. https://doi.org/10.14745/ccdr.v43i12a01f

\section{Introduction}

Le virus de l'immunodéficience humaine $(\mathrm{VIH})$ et le syndrome d'immunodéficience acquise (SIDA) sont une épidémie persistante à l'échelle internationale. Bien qu'il y ait eu d'énormes progrès dans les efforts internationaux pour mettre fin à l'épidémie de VIH/SIDA au cours des 15 dernières années (1), on estime à 2,1 millions le nombre de nouveaux (incidents) cas déclarés en 2015 (2). Malgré cela, le nombre de nouveaux cas d'infection au VIH dans les pays à revenu élevé est relativement faible, avec une moyenne de taux de diagnostic de 6,3 pour 100000 habitants pour les pays de l'Organisation de coopération et de développement économiques (OCDE) inclus dans le rapport de surveillance du VIH et du SIDA (3). Bien que le taux de nouveaux diagnostics du Canada a été inférieur à la moyenne des pays de l'OCDE, il se situe encore loin derrière 
d'autres pays comme les Pays-Bas, l'Allemagne, la Suède et la Finlande.

L'Agence de santé publique du Canada (ASPC) travaille en partenariat avec les gouvernements provinciaux et territoriaux, $d$ 'autres ministères fédéraux et des organisations non gouvernementales de lutte contre le VIH/sida au Canada, contribue aux efforts internationaux pour éliminer le VIH/SIDA et suit les progrès par le biais de l'Initiative fédérale de lutte contre le VIH/sida au Canada. La publication annuelle des données de surveillance du VIH s'effectue dans le cadre du mandat de I'ASPC qui consiste à recueillir et à analyser les données de surveillance à l'échelle nationale (4).

L'objectif de ce rapport est de fournir un aperçu descriptif de I'épidémiologie de tous les diagnostics d'infection au VIH au Canada jusqu'en 2016 par situation géographique, sexe, âge, catégorie d'exposition et race/ethnicité, ainsi que des données sur le nombre de nourrissons ayant été exposés au VIH pendant la période périnatale et la proportion de ces nourrissons recevant un traitement antirétroviral. Antérieurement, les données sur le $\mathrm{VIH}$, en combinaison avec des données de surveillance du sida, ont été publiées chaque année par l'ASPC dans un rapport distinct intitulé Le VIH et le sida au Canada Rapport de surveillance (3). II s'agit de la première version du rapport publié sous un nouveau titre dans le Rapport des maladies transmissibles au Canada (RMTC). Notez qu'un rapport de surveillance distinct pour les cas de SIDA 2016 apparaît également dans ce numéro du RMTC (5). Les données de ces rapports remplacent toutes les données déjà publiées dans la série de la surveillance du VIH et du SIDA, car elles représentent I'information la plus récente. Des informations supplémentaires, dont des tableaux de données et des définitions des catégories d'exposition, sont présentés en ligne sur un supplément Web, sur le site du RMTC (6).

\section{Méthodologie}

Les données utilisées pour préparer ce rapport de surveillance du VIH proviennent de trois sources différentes : le système national de surveillance du $\mathrm{VIH} /$ sida maintenu par I'ASPC, les données recueillies grâce au dépistage médical du VIH aux fins d'immigration par Immigration, Réfugiés et Citoyenneté Canada (IRCC), et le Programme de surveillance périnatale du VIH au Canada (PSPVC).

\section{Système de surveillance du VIH/SIDA}

Le système national de surveillance du $\mathrm{VIH} /$ sida est un système de surveillance passive basé sur les cas qui recueille des données non nominatives sur les personnes qui ont reçu un diagnostic d'infection par le VIH. Plus de détails sur la méthodologie du système national de surveillance du VIH/SIDA, y compris les processus de collecte de données, la gestion de données, le contrôle de la qualité des données, I'analyse, la classification et la catégorisation des sous-groupes de la population, ont préalablement été décrits (3). En bref, les données, incluant sans s'y limiter l'âge, le sexe, la race ou l'origine ethnique et les risques associés à la transmission du VIH (catégories d'exposition), sont volontairement soumises à l'ASPC par tous les gouvernements provinciaux et territoriaux responsables de la santé publique. Plusieurs limites sont présentes en ce qui concerne les données soumises par les provinces sur la race et l'origine ethnique, et la catégorie d'exposition.
Plus particulièrement, le Québec ne soumet pas à l'ASPC d'information sur la catégorie d'exposition ou sur la race ou I'origine ethnique concernant les cas de VIH. En ce qui a trait à l'Ontario, les renseignements sur la catégorie d'exposition étaient limités pour les cas de VIH déclarés avant 2009, et aucun renseignement sur la race ou l'origine ethnique n'était disponible pour les cas de VIH déclarés avant 2009. Concernant la Colombie-Britannique, les données sur la race ou l'origine ethnique n'ont pas été soumises pour l'année de déclaration actuelle et toutes les données historiques de l'origine ethnique ont été retirées, à la demande de la province, en attendant un examen des pratiques de présentation de ces données.

Les cas signalés à l'ASPC doivent répondre à la définition nationale de cas (7). Les provinces et les territoires fournissent des données au moyen du Formulaire de déclaration de cas à l'échelle nationale (3) ou par l'intermédiaire d'une transmission de données électronique sécurisée. Toutes les données brutes (formulaires papier et fichiers de données électroniques) sont conservées dans le respect de la directive de l'Agence sur la collecte, I'utilisation et la diffusion de l'information sur la santé publique (ASPC, 2013, document non publié). L'évaluation de la qualité des données, comme la détection de doublons, est gérée par les provinces et les territoires avant d'être soumise à I'ASPC. Les données présentées dans ce rapport de surveillance représentent des cas de $\mathrm{VIH}$ et du sida diagnostiqués le 31 décembre 2016 ou avant, et soumis à l'ASPC par les programmes de surveillance provinciaux et territoriaux jusqu'au 7 juin 2017.

Dans ce rapport de surveillance, les termes «cas» ou "cas déclarés» désignent les personnes diagnostiquées par une province ou un territoire au cours d'une année donnée et déclarées à I'ASPC. Puisque les données de surveillance ne peuvent rendre compte que de la partie diagnostiquée de l'épidémie, la modélisation et le recours à des sources additionnelles d'information sont essentiels pour dépeindre l'épidémie chez les Canadiens souffrant d'une infection au VIH ou du sida, tant diagnostiquée que non diagnostiquée (8).

\section{Dépistage médical du VIH aux fins d'immigration}

Les étrangers qui demandent un visa de résident permanent ou de résident temporaire doivent se soumettre à un examen médical aux fins de l'immigration (EMI), soit au Canada ou à l'étranger, géré par IRCC. IRCC effectue le dépistage de routine du VIH obligatoire pour tous les demandeurs de 15 ans et plus, ainsi que ceux de moins de 15 ans qui présentent certains facteurs de risque (9). IRCC fournit à l'ASPC des données non nominatives recueillies lors de l'EMI, y compris les données démographiques ainsi que l'année de tests (pour ceux qui ont été testés au Canada) ou l'année où le demandeur a été admis au Canada (pour ceux qui ont été testés à l'étranger). Les données relatives au VIH diagnostiqué au moyen d'un EMI ont été extraites en janvier 2017; toutefois, en raison de retards de la transmission des données, seules les données jusqu'à la fin de 2015 ont été fournies.

\section{Programme de surveillance périnatale du VIH au Canada}

Des données nationales sur l'état sérologique des nouveau-nés exposés en période périnatale à l'infection au VIH sont recueillies 
par l'intermédiaire du PSPVC, une initiative du Groupe canadien de recherche sur le sida chez les enfants. Le PSPVC est un système de surveillance sentinelle qui recueille des données sur tous les nourrissons et enfants nés de mères reconnues comme étant séropositives pour le VIH au Canada, y compris ceux qui sont nés à l'extérieur du Canada et traités contre une infection au VIH. Les données ont été obtenues au moyen d'une enquête nationale confidentielle anonyme sur les nourrissons connus des pédiatres travaillant dans des centres de soins tertiaires et des spécialistes du VIH œuvrant dans des cliniques spécialisées à l'échelle du pays. Des renseignements supplémentaires sur la méthodologie du PSPVC ont été décrits précédemment (3). Les données relatives à la surveillance périnatale jusqu'à la fin de 2016 présentées dans le présent rapport de surveillance ont été extraites de la base de données du PSPVC en juillet 2017.

\section{Analyse}

Les logiciels Microsoft Excel 2010 et SAS Enterprise Guide v 5.1 ont été utilisés pour le nettoyage et l'analyse des données. Des procédures normalisées d'enregistrement des données ont été appliquées à toutes les données afin de créer un ensemble de données nationales utilisé aux fins d'analyse. Aucune procédure statistique n'a été utilisée pour les analyses comparatives du présent rapport et aucune technique statistique n'a été appliquée pour tenir compte des données manquantes. II convient de noter qu'il existe différentes exigences et pratiques en matière de déclaration du VIH dans tout le pays (10) et que l'intégralité de certaines informations épidémiologiques varie entre les provinces et les territoires. Ainsi, lorsque les pourcentages ont été calculés, les données manquantes ont été exclues.

Le terme "adulte» est utilisé dans le rapport lors de l'examen de certaines variables, telles que la catégorie d'exposition. Aux fins du présent rapport, un "adulte» signifie toute personne de 15 ans ou plus.

À l'exception des cas où la suppression des données a été demandée par la province ou le territoire, les données dans les tableaux ayant des cellules de petite taille ( $n$ moins de 5 ) n'ont pas été supprimées, étant donné que la divulgation n'est pas considérée comme un risque d'identification des cas individuels. Ces procédures sont en conformité avec la directive de l'Agence sur la collecte, I'utilisation et la diffusion de l'information sur la santé publique. Les données ont été vérifiées par les provinces et les territoires pour en assurer l'exactitude. Les principaux résultats sont résumés dans ce document. Les tableaux supplémentaires peuvent être trouvés dans le supplément Web (6).

\section{Résultats}

Un total de 2344 nouveaux cas d'infection au VIH ont été signalés au Canada en 2016, soit une augmentation de 11,6\% du nombre de cas signalés en 2015 (2 100 cas). Il s'agit du plus grand nombre de cas d'infections au VIH signalés depuis 2009 (2 364 cas). Il correspond à une hausse du taux de diagnostic national, qui est passé de 5,8 pour 100000 habitants en 2015 à 6,4 pour 100000 habitants (figure 1).

Au Canada, un total cumulatif de 84409 cas d'infection au VIH a été signalé à l'ASPC depuis le début de la déclaration des cas de VIH en 1985. Une diminution régulière des cas signalés d'infection au VIH a été observée jusqu'à l'année 2000 (2 062 cas), puis une courte montée jusqu'à un plateau de 2002 à 2008, période où le nombre annuel de cas signalés d'infection au VIH a fluctué entre 2403 et 2 599. Le nombre de cas d'infections au VIH déclarés a ensuite diminué progressivement de 2008 à 2014, suivi par une augmentation en 2015 et en 2016.

Figure 1: Nombre de cas de VIH déclarés, y compris les taux de diagnostic, par année du test - Canada, 1996 à 2016

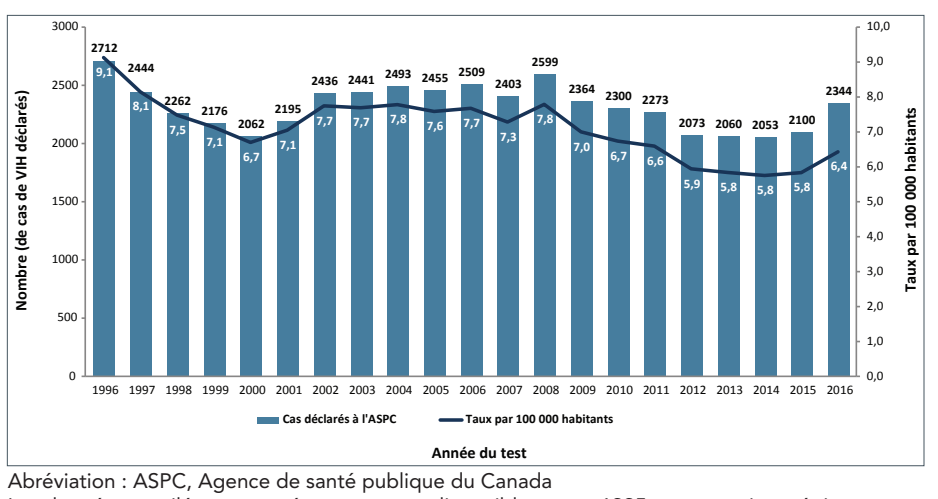

Les données ventilées par année ne sont pas disponibles avant 1995 pour certaines régions

\section{Distribution géographique}

En 2016, I'Ontario représentait la plus forte proportion et le plus grand nombre de cas de VIH signalés ( $n=881,37,6 \%$ ), suivi par le Québec ( $n=593,25,3 \%$ ) et l'Alberta $(n=282,12,0 \%)$.

Les taux de diagnostics de VIH au niveau provincial et territorial révèlent des variations notables à l'échelle du pays. En 2016, la Saskatchewan représentait $7,4 \%(n=174)$ du total des nouveaux cas d'infections au VIH déclarés et a continué d'avoir le plus haut taux de diagnostic de l'infection au VIH à 15,1 pour 100000 habitants, soit plus du double de celui de l'ensemble de la population canadienne. Le Manitoba, représentant 5,4 \% ( $n=126$ ) des cas d'infections au VIH en 2016, avait le deuxième plus haut taux de diagnostic d'infection au VIH à 9,5 pour 100000 habitants, suivi par le Québec (7,1 pour 100000 habitants) et l'Alberta (6,6 pour 100000 habitants). Les taux de diagnostic dans toutes les autres provinces et tous les autres territoires étaient inférieurs au taux national de 6,4 pour 100000 habitants (figure 2, tableaux supplémentaires 1-3) (6).

Figure 2 : Taux de diagnostic de VIH (pour 100000 habitants) selon la province ou le territoire - Canada, 2016

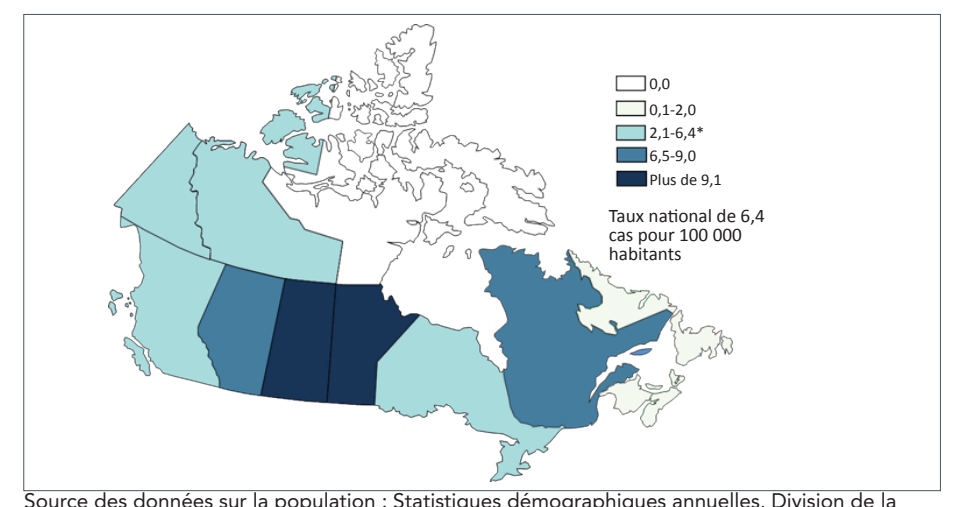
démographie, Statistique Canada, octobre 2016 


\section{Distribution selon le groupe d'âge et le sexe}

Les données sur les groupes d'âge ont été disponibles pour $99,9 \%$ des cas de VIH déclarés pour 2016. Depuis 1985 au Canada, 37,2\% des cas signalés d'infection au VIH ont été diagnostiqués chez les personnes ayant de 30 à 39 ans. En 2016, les personnes de 30 à 39 ans continuent de représenter le groupe comptant le plus de nouveaux cas de $\mathrm{VIH}(28,7 \%)$, mais avec une proportion plus faible que les années précédentes. Les cas des personnes ayant de 30 à 39 ans ont connu la plus forte augmentation de 2015 à 2016 à 17,3\%, suivis de près par ceux de 50 ans ou plus, à $13,4 \%$.

Au cours des cinq dernières années, la plus forte augmentation en ce qui a trait à la proportion de cas de VIH par âge a été observée dans les cas des personnes de 50 ans et plus, avec une augmentation de 6,6\% de 2012 à 2016 (17,7 \% à 24,3\%). La plus forte baisse a été constatée chez les personnes ayant de 40 à 49 ans, avec une diminution de 5,8\% de 2012 à 2016 $(27,8 \%$ à $22,0 \%)$. Les jeunes de 15 à 19 ans représentaient $2,1 \%$ des cas en 2016. Ce groupe a connu une augmentation faible mais régulière du nombre de cas d'infections au $\mathrm{VIH}$ signalés au cours des cinq dernières années, alors que les cas d'infections au VIH signalés chez les enfants (moins de 15 ans) sont demeurés assez constants au cours de la même période par rapport à tous les autres groupes d'âge (figure 3, tableaux supplémentaires 3,6$)(6)$.

Les données sur le sexe étaient disponibles pour $99,6 \%$ des cas de VIH signalés en 2016. Au Canada, les hommes ont toujours représenté un pourcentage plus élevé de cas de $\mathrm{VIH}$ signalés que les femmes. Au cours de la dernière décennie, la proportion annuelle des cas de VIH déclarés chez les adultes (15 ans et plus) est demeurée stable, à environ un quart des nouveaux cas. Cette tendance a également été observée en 2016 avec les femmes, qui représentaient $23,3 \%$ de tous les cas signalés (tableaux supplémentaires 2, 4-6) (6).
Figure 3: Proportion des cas de VIH signalés par groupe d'âge et année du test - Canada, 2007 à 2016

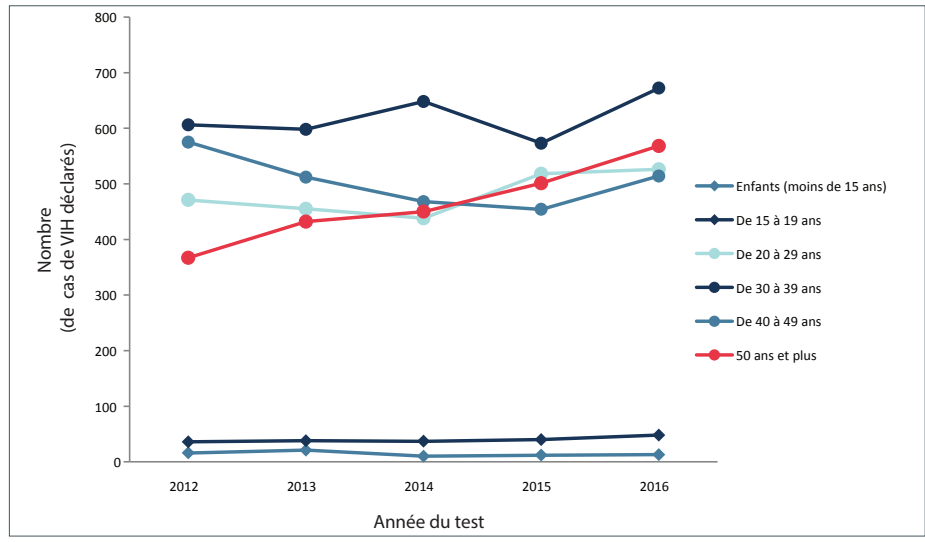

Au cours des dernières années, une augmentation de la proportion des cas de VIH dans les groupes plus âgés pour les deux sexes a été constatée. Cette tendance s'est poursuivie en 2016, avec une large proportion de cas de personnes de 50 ans et plus pour les deux sexes, soit $25,6 \%$ chez les hommes et $20,3 \%$ chez les femmes (figure 4). Il y a également une évolution au fil du temps dans la distribution selon l'âge pour les deux sexes. Historiquement, bien que les cas de VIH aient été plus fréquents dans les groupes d'âge plus jeunes chez les femmes et dans les groupes d'âge plus âgés chez les hommes, il y a eu une diminution de $11,3 \%$ chez les femmes ayant de 20 à 29 ans et une augmentation de 7,5\% dans le groupe d'âge masculin correspondant depuis 2001. Chez les deux sexes, les cas des personnes ayant de 30 à 39 ans représentaient encore la plus forte proportion de cas de $\mathrm{VIH}$ avec $27,7 \%$ et $31,8 \%$ d'hommes et de femmes respectivement en 2016 (figure 4).

\section{Figure 4 : Distribution des cas de VIH déclarés selon le groupe d'âge et le sexe - Canada, 2016}

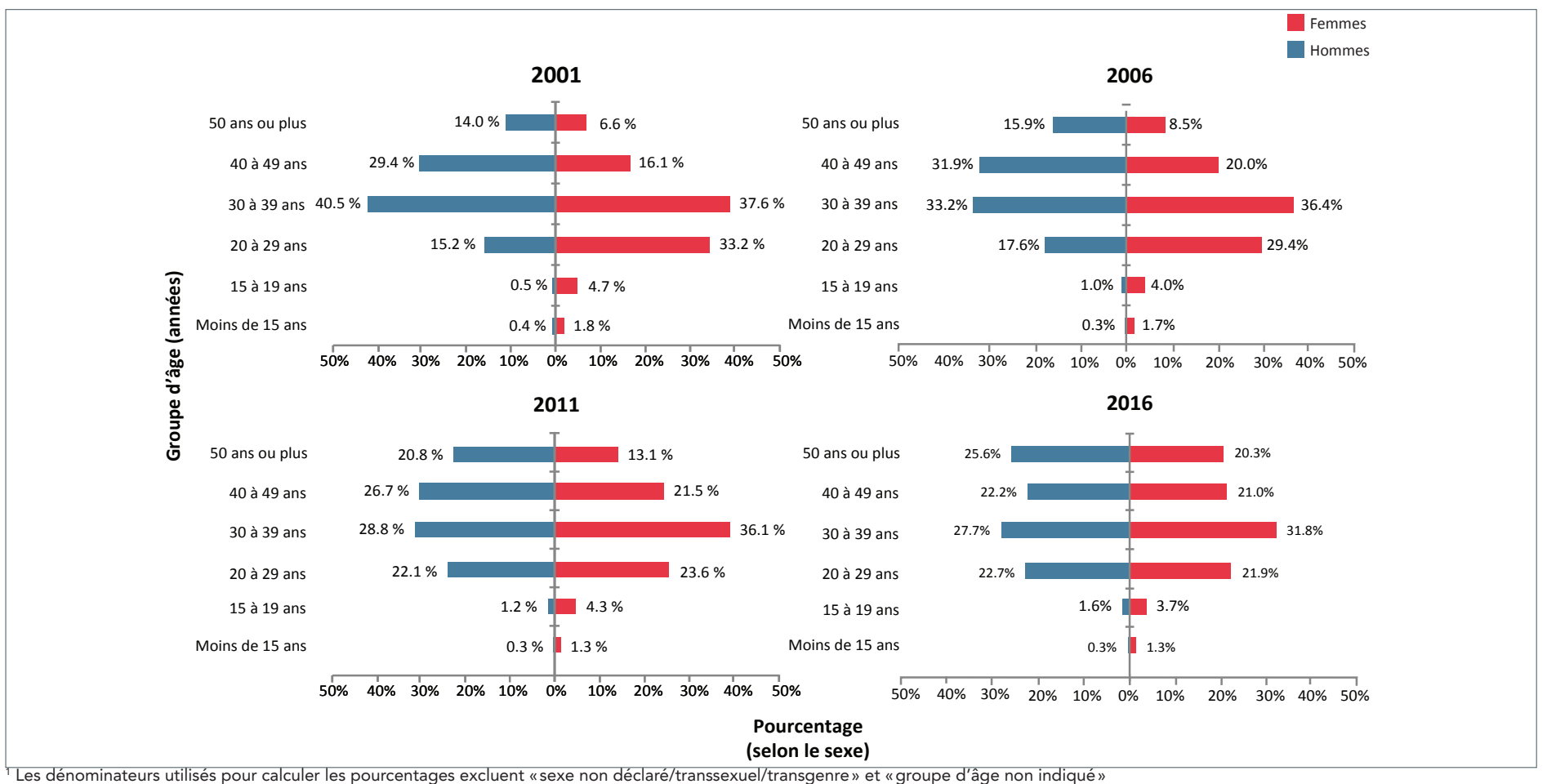




\section{Distribution par catégorie d'exposition}

La catégorie d'exposition des hommes ayant des relations sexuelles avec d'autres hommes (HARSAH) a continué à représenter le plus grand nombre et la plus grande proportion de tous les cas déclarés d'infection au VIH chez les adultes. En 2016, chez les adultes dont la catégorie d'exposition était connue $(61,6 \%$ de tous les cas), un peu moins de la moitié $(44,1 \%)$ a été attribuée à la catégorie d'exposition des HARSAH. La deuxième catégorie d'exposition signalée étant la plus élevée était celle des contacts hétérosexuels (32,3\%), avec une assez bonne répartition des cas de $\mathrm{VIH}$ attribués à un contact hétérosexuel avec une personne originaire d'un pays où le $\mathrm{VIH}$ est endémique (Hét-endemique, 10,5\%), le contact hétérosexuel avec une personne à risque (Hét-risque, $8,9 \%$ ) et le contact hétérosexuel sans aucun risque signalé (ARS-Hét, 12,9\%). La troisième catégorie d'exposition la plus souvent signalée chez les adultes était celle de l'utilisation de drogues injectables (UDI), représentant 15,1\% des cas de VIH signalés (figure 5, tableau supplémentaire 7) (6). Aucun changement significatif dans les proportions de catégories d'exposition n'a été relevé entre 2015 et 2016.

Figure 5 : Proportion des cas de VIH déclarés parmi les adultes (15 ans et plus), hommes et femmes adultes par catégorie d'exposition - Canada, 2016 $16^{1,2}$

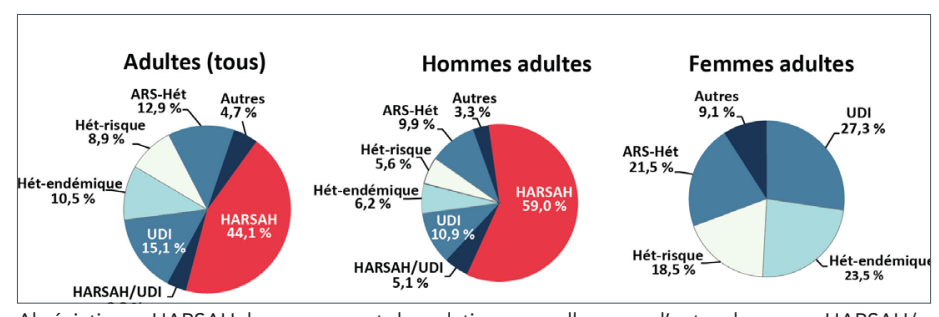

Abréviations : HARSAH, hommes ayant des relations sexuelles avec d'autres hommes, HARSAH/ UDI, hommes ayant des relations sexuelles avec d'autres hommes et utilisant des drogues injectables; UDI, utilisation de drogues injectables; Hét-endémique, contact hétérosexuel avec une personne originaire d'un pays où le $\mathrm{VIH}$ est endémique; Hét-risque, contact hétérosexuel avec une personne à risque; ARS-Hét, contact hétérosexuel sans aucun risque signalé ${ }^{1}$ Les pourcentages sont fondés sur le nombre total de cas déclarés, à l'exclusion des «ARS» et des cas «non déclarés». Notez que les données sur les catégories d'exposition n'étaient pas disponibles pour le Québec. Les chartes des hommes et des femmes excluent les cas d'infections au VIH où le sexe n'a pas été déclaré ou déclaré comme transgenre ou transsexuel ${ }^{2}$ "Autres» comprend les receveurs de sang ou de facteurs de coagulation, les voies d'exposition non spécifiées répertoriées comme "Autres» et de l'Alberta, les cas considérés comme "hors du pays»

La distribution des cas de VIH parmi les hommes et femmes adultes varie selon la catégorie d'exposition. En 2016, la catégorie d'exposition des HARSAH a continué de représenter la plus grande proportion $(59,0 \%)$ des cas de VIH déclarés chez les hommes adultes, suivie de tous types de contacts hétérosexuels $(21,6 \%)$ et de I'UDI (10,9\%). Chez les femmes adultes, les contacts hétérosexuels représentaient 63,5\% des cas déclarés (23,5 \% Hét-endémique, 21,5\% ARS-Hét et $18,5 \%$ Hét-risque, suivi par I'UDI 27,3\%). En ce qui a trait aux rapports hétérosexuels, il y avait une différence importante entre les hommes et les femmes dans la catégorie d'exposition Hét-endémique. Les cas de la catégorie Hét-endémique signalés chez les hommes représentaient 6,2\%, comparativement à $23,5 \%$ chez les femmes. La catégorie d'exposition des UDI représentait un peu plus d'un quart des cas de $\mathrm{VIH}$ chez les femmes adultes (27,3\%), comparativement à $16,0 \%$ des cas de VIH chez les hommes adultes (10,9\% par exposition à I'UDI et jusqu'à $5,1 \%$ dans la catégorie d'exposition des HARSAH/UDI (figure 5, tableaux supplémentaires 8-11) (6).

\section{Distribution selon la race ou l'origine ethnique}

En 2016, les renseignements sur la race ou l'origine ethnique étaient disponibles pour presque la moitié $(48,6 \%)$ des cas de VIH déclarés. Depuis 1998, au moment où les données sur la race ou l'origine ethnique ont été recueillies pour la première fois, la race ou l'origine ethnique caucasienne représentait la plus grande proportion de nouveaux cas de VIH au Canada, pour tous les âges et tous les sexes (44,4 \%). En 2016, moins de la moitié des cas de $\mathrm{VIH}$ ayant une race ou une origine ethnique connue étaient de race caucasienne $(40,4 \%)$, suivie par la race afro-américaine $(21,9 \%)$ et les populations autochtones $(21,2 \%)$. La race ou l'origine ethnique autochtone se composait comme suit : $19,0 \%$ de membres des Premières Nations, 1,6\% de Métis, 0,4 \% d'Autochtones d'origine non précisée et 0,3\% d'Inuits. Entre 2015 et 2016, c'est le sous-groupe des Premières Nations qui a enregistré la plus grande progression $(29,9 \%)$ dans le nombre des cas par rapport aux autres races et origines ethniques et aux autres sous-groupes autochtones (figure 6, tableau supplémentaire 12) (6).

Figure 6 : Proportion de cas de VIH déclarés (tous âges confondus) par race ou origine ethnique (incluant les sous-groupes autochtones) - Canada, 2016 (1,2,3,4 $^{2}$

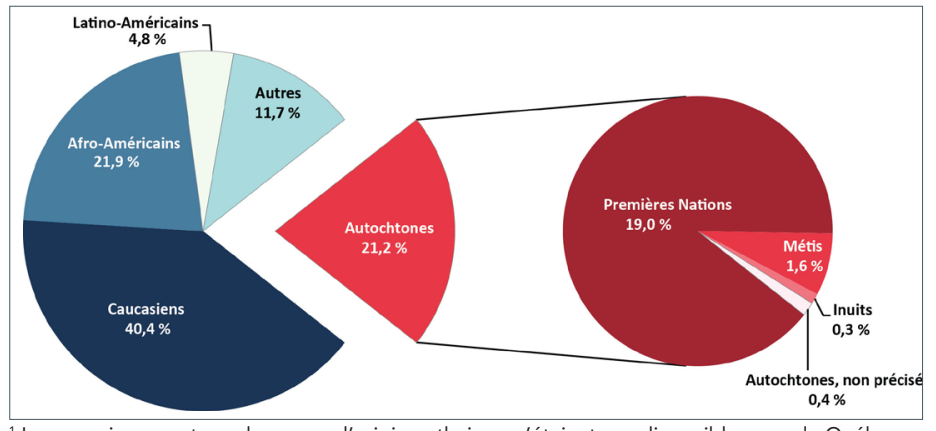

'Les renseignements sur la race ou l'origine ethnique n'étaient pas disponibles pour le Québec et la Colombie-Britannique

2 Exclut les cas où la race ou l'origine ethnique n'a pas été déclarée

Les "Latino-Américains » comprennent, par exemple, Mexicains, Centraméricains et Sud-Américains

${ }^{4}$ Les «Autres» comprennent, par exemple, Pakistanais, Sri-Lankais, Bangladais, Arméniens, Égyptiens, Iraniens, Libanais, Marocains, Chinois, Japonais, Vietnamiens, Cambodgiens, Indonésiens, Laotiens, Coréens, Philippins, Somaliens, Haïtiens et Jamaïcains

Des variations dans la distribution selon le sexe de la race ou de l'origine ethnique ont également été observées. En 2016 , la majorité des cas signalés l'ont été chez les hommes de race ou d'origine ethnique caucasienne (47,8\%), suivie par la race afro-américaine $(16,4 \%)$ et les populations autochtones $(15,4 \%)$. Par comparaison, un peu plus du tiers des femmes étaient afro-américaines (36,5\%), suivies par les femmes autochtones $(36,2 \%)$ et caucasiennes $(21,0 \%$ ) (figure 7 , tableaux supplémentaires 13,14$)(6)$. 
Figure 7 : Proportion de cas de VIH déclarés (tous âges confondus) selon la race ou l'origine ethnique - Canada, $2016^{1,2,3,4}$

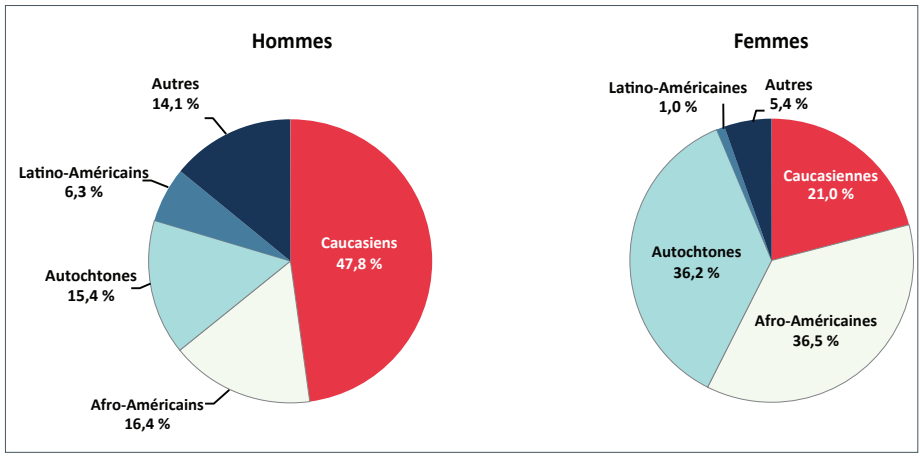

Les renseignements sur la race ou l'origine ethnique ne sont pas disponibles pour le Québec et la Colombie-Britannique

Les dénominateurs utilisés pour calculer les pourcentages excluent les cas d'infection au VIH où la race ou l'origine ethnique était "non indiquée » et les cas d'infection au VIH où le sexe était "non indiqué» ou déclare comme "transsexuel ou transgenre

${ }^{3}$ Les Latino-Américains comprennent, par exemple, Mexicains, Centraméricains et Sud-Américains

Les autres comprennent, par exemple, Pakistanais, Sri-Lankais, Bangladais, Arméniens, Eyptiens, Iraniens, Libanais, Marocains, Chinois, Japonais, Vietnamiens, Cambodgiens, Indonesiens, Laotiens, Coreens, Philippins, Somaliens, Haitiens et Jamaicains

\section{Distribution des cas de VIH déclarés selon la catégorie d'exposition et selon la race ou l'origine ethnique}

De l'information sur la race ou l'origine ethnique et sur la catégorie d'exposition était disponible pour $47,4 \%$ des cas signalés en 2016. En 2016, la majorité des cas ont été signalés comme étant de race caucasienne dans la catégorie d'exposition des HARSAH (56,9\%). Des cas attribuables à I'UDI, la quasi-totalité des cas de VIH ont été soit chez les Autochtones $(59,6 \%)$ ou les Caucasiens $(37,7 \%)$. Le principal groupe ethnique ou racial parmi les cas déclarés attribués au contact hétérosexuel était la race afro-américaine $(41,7 \%), 84,3 \%$ de ses membres ont été déclarés dans la catégorie d'exposition Hét-endémique (figure 8, tableau supplémentaire 15) (6).

Figure 8 : Proportion de cas de VIH déclarés (tous âges confondus) selon la catégorie d'exposition et selon la race ou l'origine ethnique - Canada, 2016 1,2,3,4,5

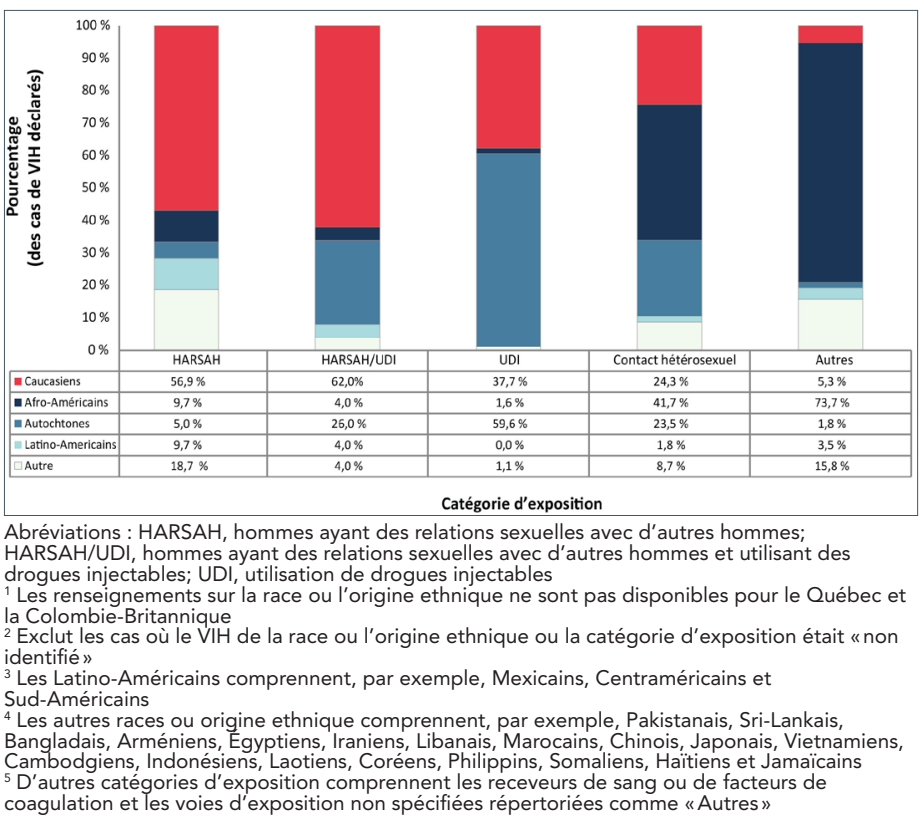

\section{Dépistage médical du VIH aux fins d'immigration}

En 2015, il y avait 550 demandeurs de statut de résident canadien qui ont été testés positifs pour le VIH. Des 550 demandeurs, 350 ont fait l'objet d'un EMI au Canada et 200 ont fait l'objet d'un EMI à l'étranger. Notez que les données ne permettent pas de distinguer entre ceux qui se sont finalement établis au Canada et ceux ne l'ayant pas fait (figure 9, tableau supplémentaire 16) (6).

Figure 9 : Distribution des demandeurs d'immigration séropositifs selon l'emplacement et l'année du test, 2005-2014 ${ }^{1,2}$

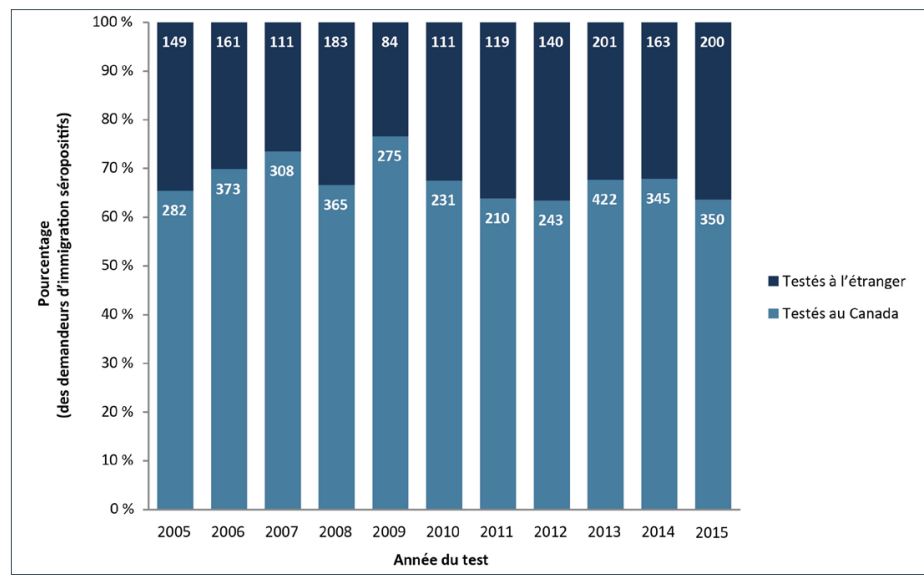

Immigration, Réfugiés et Citoyenneté Canada, Base de données sur le VIH de l'IRCC en date de juillet 2017. Immigration, Réfugiés et Citoyenneté Canada, Base de données des avis provinciaux et étrangers de l'Unité de liaison avec les autorités de la santé publique relatifs aux évaluations de l'état de santé après l'arrivée de la Direction générale de la santé d'IRCC, en date de juillet 2017. Reproduit et distribué avec l'autorisation d'Immigration, Réfugiés et Citoyenneté Canada 2 Pour les demandeurs testés au Canada, l'année se réfère à l'année du test. Pour les demandeurs testés à l'étranger, l'année se réfère à l'année où le demandeur s'est établi au Canada

Parmi les candidats dépistés au Canada entre 2002 et 2015, 4364 personnes au total ont reçu un diagnostic d'infection au $\mathrm{VIH}$, avec une moyenne de 312 par année (allant de 210 à 422). Au total, 57,1 \% de ces demandeurs séropositifs étaient de sexe masculin. La majorité des candidats dépistés au Canada avait de 30 à 39 ans (41,9\%) ou de 40 à 49 ans (25,0\%). La proportion la plus élevée de demandeurs séropositifs pour le VIH se trouvait en Ontario $(54,7 \%)$, suivie par le Québec $(25,2 \%)$, la Colombie-Britannique (8,7 \%) et l'Alberta $(7,6 \%)$ (tableau supplémentaire 17) (6).

Parmi les demandeurs dépistés à l'étranger entre 2005 et 2015 qui se sont établis au Canada, 1622 ont reçu un diagnostic d'infection au VIH, à une moyenne de 147 par année (allant de 84 à 201). Un peu plus de la moitié $(51,4 \%)$ était des femmes. La majorité des cas $(40,0 \%)$ avaient de 30 à 39 ans, suivie par ceux qui avaient de 20 à 29 ans (25,3\%). L'Ontario était la province de résidence la plus souvent visée $(33,4 \%)$, suivie par le Québec (25,0 \%), l'Alberta (14,4\%), la Colombie-Britannique (12,9\%) et le Manitoba (8,7\%). Parmi les demandeurs séropositifs, $72,1 \%$ étaient nés dans des pays où le $\mathrm{VIH}$ est endémique (tableau supplémentaire 17) (6).

\section{Programme de surveillance périnatale du VIH au Canada}

Entre 1984 et 2016, au Canada, on a déterminé que

4849 nourrissons ont été exposés au VIH pendant la période 
périnatale. Le nombre déclaré de nourrissons exposés au VIH, selon l'année de naissance, a augmenté entre 2009 et 2016, passant de 200 en 2009 à un pic de 263 en 2016. (figure 10, tableaux supplémentaires 18-22) (6).

Figure 10 : Nombre de nourrissons exposés au VIH durant la période périnatale et proportion de nourrissons exposés au VIH durant la période périnatale recevant un traitement antirétroviral en période périnatale selon l'année de naissance - Canada, 2009 à 2016

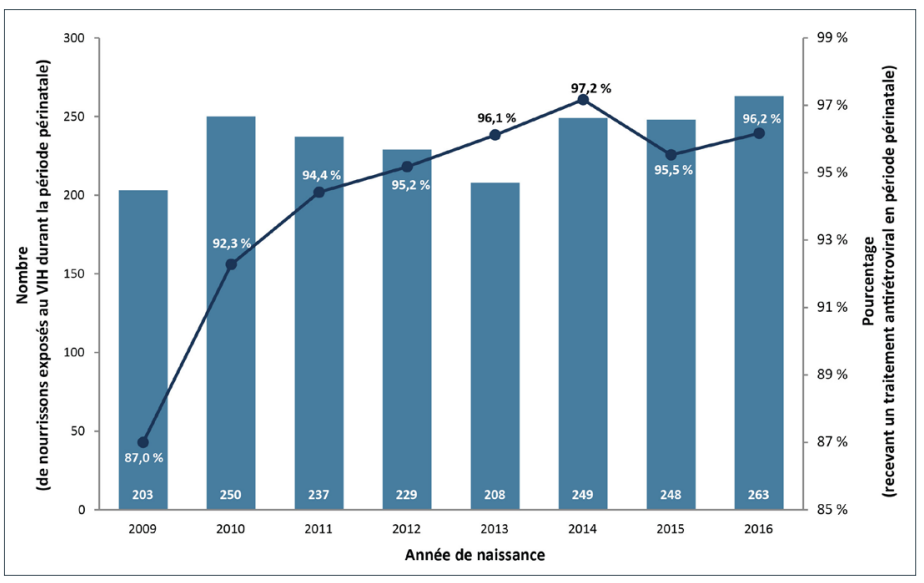

Abréviations : TA, traitement antirétroviral

Des 263 nourrissons exposés à une transmission périnatale nés en 2016, une transmission du VIH a été confirmée chez une mère qui ne recevait aucun traitement antirétroviral périnatal. Le pourcentage de mères séropositives pour le $\mathrm{VIH}$ recevant un traitement antirétroviral (a augmenté au fil du temps et s'est stabilisé depuis 2011, variant entre 94,4\% (2011) et 97,2 \% (2014); en 2016, le pourcentage était légèrement inférieur à celui de 2014 à 96,2 \% (figure 10, tableau supplémentaire 18) (6).

Un examen de tous les nourrissons ayant été exposés pendant la période périnatale entre 1984 et 2016 indique que, pour 73,8 \% d'entre eux, l'infection de la mère était attribuable à des contacts hétérosexuels (71,0\% en 2016) et, pour $22,8 \%$, elle était attribuable à I'UDI (22,3\% en 2016) (tableau supplémentaire 19) (6).

En ce qui concerne la distribution selon la race ou l'origine ethnique chez les nourrissons ayant été exposés pendant la période périnatale entre 1984 à 2016, 49,8\% ont été identifiés comme Afro-Américains, 23,8 \% comme Caucasiens et 17,8 \% comme Autochtones. En 2016, la race afro-américaine est restée la race ou l'origine ethnique la plus souvent signalée, avec une proportion (48,7\%) similaire à celle observée en 1984 à 2016. La proportion d'enfants des peuples de race autochtone a progressivement augmenté, pour atteindre un sommet de $25,5 \%$ en 2016 , et la proportion d'enfants de race caucasienne a diminué à un creux de 11,8 \% (tableau supplémentaire 20) (6).

La région de naissance de la mère pour la majorité des nourrissons entre 1984 et 2016 était l'Amérique du Nord (42,4 \%), I'Afrique $(38,2 \%)$ et les Caraïbes $(9,8 \%$ ) (tableau supplémentaire 21). Pour la même période, la plus forte proportion de nourrissons exposés au VIH pendant la période périnatale a été signalée en Ontario (34,6\%), suivie du Québec $(25,7 \%)$ et de l'Alberta (14,1\%) (tableau supplémentaire 22) (6).

\section{Discussion}

En 2016, il y a eu une augmentation de 11,6\% du nombre de diagnostics de $\mathrm{VIH}$ par rapport à 2015, ce qui représente le taux le plus élevé depuis 2011. Un certain nombre de possibilités existent pour expliquer cette augmentation du nombre de cas, y compris une augmentation du dépistage en raison de la mise en œuvre des initiatives provinciales d'évaluation (11-13).

La majorité des augmentations a été observée au Québec, en Alberta, au Manitoba et en Ontario, bien que, globalement, la Saskatchewan avait le plus haut taux absolu. Pour ce qui est de l'âge, du sexe et de la race ou de l'origine ethnique, les plus fortes augmentations ont été observées respectivement chez les personnes ayant de 30 à 39 ans, l'homme adulte et les membres des Premières Nations. Il est également important de noter que, pour le Québec, l'augmentation s'explique en partie par un passage partiel en 2016 à des tests nominaux, en partant de tests non nominaux, car les cas nominatifs n'ont pas tous été saisis aux fins de présentation au niveau fédéral.

Au niveau national, les différences observées précédemment entre la répartition par âge des hommes et des femmes ont été réduites au cours des cinq dernières années, d'où une distribution d'âge similaire entre les sexes pour 2016. Cet effet est dû à l'augmentation des proportions diagnostiquées dans les groupes d'âge pour les deux sexes. La proportion de diagnostics de VIH parmi les Canadiens de 50 ans et plus a augmenté graduellement depuis 1985. II est possible que le changement observé ait été entraîné par une population ayant des facteurs de risque spécifiques, comme une plus forte proportion de personnes veuves et divorcées entreprenant une nouvelle relation, de plus faibles proportions de relations sexuelles protégées en raison de l'absence de préoccupations liées à la grossesse et une plus forte stigmatisation de la discussion des habitudes sexuelles et de la prise de drogues chez les médecins de ce groupe plus âgé (14).

Au niveau national, des différences continuent à être observées entre les hommes et les femmes selon la distribution en fonction de la catégorie d'exposition, et la race ou l'origine ethnique Les hommes qui ont des rapports sexuels avec des hommes, la catégorie d'exposition la plus fréquente chez les hommes, était associée à la race caucasienne, tandis que les contacts hétérosexuels et I'UDI, les deux catégories d'exposition les plus fréquentes chez les femmes, étaient respectivement associées aux personnes afro-américaines et autochtones. Si la race caucasienne comptait le taux le plus élevé, les Autochtones et les Afro-Américains étaient surreprésentés alors qu'ils représentent moins de $5 \%$ de la population au Canada $(15,16)$, mais représentent individuellement plus de $20 \%$ des nouveaux cas signalés d'infection au VIH. Toutefois, il convient de noter que les données sur l'origine ethnique n'étaient disponibles que dans environ la moitié des cas déclarés.

Les tendances dans la catégorie d'exposition ont évoluées depuis le début de la déclaration des cas d'infections au $\mathrm{VIH}$ en 1985. Au début de l'épidémie, plus de $80 \%$ de tous les cas de VIH déclarés par catégories d'exposition connues ont été attribués aux HARSAH. Bien que cette catégorie d'exposition soit encore prédominante au Canada, la proportion a diminué 
au cours des années. Les expositions aux contacts hétérosexuels et à l'UDI sont demeurées respectivement les deuxième et troisième catégories prédominantes de l'exposition au VIH au Canada.

\section{Points forts et limites}

Le VIH au Canada - Rapport de surveillance, 2016 est la principale source de données nationales concernant les nouveaux cas d'infections au VIH déclarés au Canada. Les données contenues dans ce rapport fournissent des renseignements pour l'élaboration et l'évaluation de politiques et de programmes, ainsi que des interventions en santé publique. Bien que les détails concernant les limites des données du système national de surveillance du VIH/ sida aient déjà été publiés (3), plusieurs limites doivent être soulignées. Premièrement, les données de ce rapport sont considérées comme provisoires et peuvent être assujetties à des changements dans les futurs rapports de surveillance du $\mathrm{VIH}$, lorsque les données seront mises à jour. Les différences entre les données publiées dans le présent rapport et les données publiées dans les rapports de surveillance provinciaux et territoriaux peuvent s'expliquer par des retards de déclaration ou des différences relatives à la date à laquelle les données ont été extraites des bases de données de surveillance provinciales et territoriales. Lorsque ces différences sont présentes, on recommande d'utiliser les données provenant des rapports provinciaux et territoriaux.

Deuxièmement, le système national de surveillance du VIH/ sida est un système de surveillance passive basé sur les cas qui recueille chaque année des données transmises volontairement à I'ASPC, à partir de toutes les autorités provinciales et territoriales de santé publique, par opposition à la sollicitation active de cas. Par conséquent, il est difficile de déterminer si toutes les personnes ayant une infection au VIH ont été identifiées et signalées. L'exactitude des données est en partie tributaire des rapports produits en temps opportuns et des mises à jour des provinces et territoires à l'ASPC. Un certain degré de décalage se produit, ce qui crée un retard de déclaration. En outre, la déclaration des cas d'infections au $\mathrm{VIH}$ pour les personnes de moins de deux ans et celles diagnostiquées avant leur immigration varie également selon les provinces et les territoires. Il peut parfois y avoir des changements de pratiques de comptes rendus provinciaux, ce qui peut influer sur les données.

Troisièmement, il est difficile de reconnaître et de supprimer les enregistrements en double des nouveaux diagnostics en raison de la nature non nominative de la déclaration des cas d'infections au VIH dans certaines régions. Dans la mesure du possible, les provinces et les territoires examinent et évaluent I'inclusion de déclarations en double afin de présenter un tableau aussi exact que possible du nombre de nouvelles personnes séropositives. Certaines provinces (p. ex., le Québec) adoptent une approche prudente pour l'élimination des doublons potentiels, y compris l'exclusion des résultats de tests anonymes. Pour les régions qui utilisent cette approche, les données présentées dans ce rapport reflètent le nombre minimal de nouveaux diagnostics de VIH dans cette région.

Quatrièmement, dans l'ensemble des provinces et des territoires, il n'existe aucune approche standard pour le traitement des cas de $\mathrm{VIH}$ diagnostiqués à l'extérieur du pays ou à l'extérieur de la province ou du territoire. Certaines provinces et territoires les considèrent comme de nouveaux cas et d'autres les excluent. En outre, des approches différentes sont également appliquées pour les diagnostics de $\mathrm{VIH}$ pour les enfants de moins de deux ans. En raison de ces deux situations, les brèves surdéclarations ou sous-déclarations des nouveaux diagnostics peuvent se produire, selon les règles provinciales ou territoriales.

Enfin, il y a plusieurs limites associées à la déclaration des catégories d'exposition et de la race ou de l'origine ethnique. Comme on l'a fait remarquer, peu ou pas de renseignements sur les catégories d'exposition ou la race et l'origine ethnique pour les cas d'infection au VIH sont soumis par le Québec et l'Ontario, et aucun renseignement sur la race ou l'origine ethnique n'est soumis par la Colombie-Britannique. En conséquence, les tendances présentées dans ce rapport peuvent ne pas être pleinement représentatives et doivent être interprétées avec prudence.

\section{Conclusion}

En 2016, le Canada a connu une augmentation du nombre et du taux de cas d'infections au VIH. Bien que ce taux de diagnostic soit inférieur à celui de toutes les années antérieures à 2012, il demeure le plus élevé des cinq dernières années. Un complément d'enquête et des données supplémentaires sont nécessaires pour déterminer l'importance et les causes de cette augmentation. L'ASPC continuera de recueillir et de publier les données de surveillance du $\mathrm{VIH}$ afin d'observer les tendances et de surveiller les progrès réalisés vers l'objectif de réduire le fardeau de l'infection au VIH au Canada et à l'étranger.

\section{Déclaration des auteurs}

A.C.B. - conceptualisation, rédaction - ébauche originale, examen et révision, supervision

M.E. - méthodologie, rédaction - ébauche originale, examen et révision, logiciels, données, validation, conservation des données, visualisation

L.J. - logiciels, validation des données, conservation des

données, écriture - examen et révision

A.A. - rédaction - ébauche originale, visualisation

O.V. - rédaction - examen et révision, visualisation

W.S. - rédaction - examen et révision, administration du projet, supervision

\section{Conflit d'intérêt}

Aucun.

\section{Remerciements}

L'ASPC tient à remercier les personnes suivantes des programmes de $\mathrm{VIH} /$ sida à l'échelle provinciale et territoriale pour leur contribution et leur participation :

Sumina Fathima, Alberta Health

Rosa Maheden, Alberta Health

Mariam Osman, Alberta Health

Ari Bitnun, Programme de surveillance périnatale du VIH au Canada 
Laura Sauvé, Programme de surveillance périnatale du VIH au Canada

Claire Lafrance, Immigration, Refugiés et Citoyenneté Canada Jacklyn Quinlan, Immigration, Refugiés et Citoyenneté Canada Clinical Prevention Service, Surveillance et épidémiologie, Centre de contrôle des maladies de la ColombieBritannique Carla Loeppky, ministère de la Santé du Manitoba

Rita RaaFat Gad, ministère de la Santé du Nouveau-Brunswick Shelley Landsburg, ministère de la Santé du Nouveau-Brunswick Sophie Wertz, ministère de la Santé du Nouveau-Brunswick Susan Earles, Health and Community Services, Population Health Branch, Terre-Neuve-et-Labrador

Jennifer Phillips, Health and Community Services, Population Health Branch, Terre-Neuve-et-Labrador

Heather Hannah, Santé et Services sociaux, Territoires du Nord-Ouest

Angela Fitzgerald, ministère de la Santé et du Mieux-être de la Nouvelle-Écosse

Angie Mullen, ministère de la Santé du Nunavut

Elaine Randell, ministère de la Santé du Nunavut

Abigail Kroch, Réseau ontarien de traitement du VIH

Juan Liu, Santé publique Ontario

Alex Marchand-Austin, Santé publique Ontario

Doug Sider, Santé publique Ontario

Michael Whelan, Santé publique Ontario

Marguerite Cameron, ministère de la Santé et du Mieux-être de

l'île-du-Prince-Édouard

Stacey Burns, ministère de la Santé et du Mieux-être de

I'Île-du-Prince-Édouard

Raphaël Bitera, Direction des risques biologiques et de la santé

au travail, Institut national de santé publique du Québec

Micheline Fauvel, Laboratoire de santé publique du Québec,

Institut national de santé publique du Québec

Maureen Hastie, Laboratoire de santé publique du Québec,

Institut national de santé publique du Québec

Raymond Parent, Direction des risques biologiques et de la santé au travail, Institut national de santé publique du Québec

Diane Sylvain, Laboratoire de santé publique du Québec, Institut national de santé publique du Québec

Helen Bangura, ministère de la Santé de la Saskatchewan

John Manalo, ministère de la Santé de la Saskatchewan

Lori Strudwick, Centre de lutte contre les maladies transmissibles du Yukon

Rachelle Wallace, Centre de lutte contre les maladies transmissibles du Yukon

Sabrina Plitt, Agence de la santé publique du Canada (Alberta)

Elsie Wong, Agence de la santé publique du Canada

(Colombie-Britannique)

Suresh Khatkar, Agence de la santé publique du Canada

(Manitoba)

Marie LaFreniere, Agence de la santé publique du Canada

(Nouvelle-Écosse)

Ashleigh Sullivan, Agence de la santé publique du Canada

(Ontario)

\section{Financement}

Ce projet a été financé par l'Agence de santé publique du Canada dans le cadre de son mandat de base.

\section{Références}

1. UNAIDS. Prevention Gap Report, Joint United Nations Programme on HIV/AIDS, Geneva, 2016. http://www.unaids. org/sites/default/files/media_asset/2016-prevention-gapreport_en.pdf

2. World Health Organization. World Health Statistics 2017: monitoring health for the SDGs, Sustainable Development Goals, Geneva, 2017. http://www.who.int/gho/publications/ world_health_statistics/2017/en/

3. Agence de la santé publique du Canada. Le VIH et le sida au Canada - Rapport de surveillance en date du 31 décembre 2014, Ottawa, 2015. https://www.canada.ca/fr/santepublique/services/publications/maladies-et-affections/vih-etsida-canada-rapport-surveillance-31-decembre-2014.html

4. Agence santé de la publique du Canada. Renforcer I'intervention fédérale dans la réponse du Canada au VIH/ sida. Le 13 août 2012 https://www.canada.ca/fr/santepublique/services/vih-sida/initiative-federale-lutte-contre-vihsida-canada/renforcer-intervention-federale-reponse-canadavih-sida/vih-sida/initiative-federale-lutte-contre-vih-sidacanada.html. [Consulté le 21 juil 2017].

5. Jonah L, Bourgeois AC, Edmunds M, Awan A, Varsaneux O, Siu W. Le sida au Canada - Rapport de surveillance 2016. Relevé des maladies transmissibles au Canada. 2017;43(12):292-7. https://canada-preview.adobecqms. net/fr/sante-publique/services/rapports-publications/ releve-maladies-transmissibles-canada-rmtc/numeromensuel/2017-43/rmtc-volume-43-12-7-decembre-2017/ sida-canada-2016.html

6. Bourgeois AC, Edmunds M, Awan A, Jonah L, Varsaneux O, Siu W. Le VIH au Canada-Tableaux supplémentaires, 2016 (Sur le Web seulement). Relevé des maladies transmissibles au Canada. 2017;43(12). https://www.canada.ca/fr/santepublique/services/rapports-publications/releve-maladiestransmissibles-canada-rmtc/numero-mensuel/2017-43/ rmtc-volume-43-12-7-decembre-2017/vih-2016-tableauxsupplementaires.html

7. Agence de la santé publique du Canada. Définitions nosologiques des maladies transmissibles faisant l'objet d'une surveillance nationale - 2009. Relevé des maladies transmissibles au Canada. 2009;35(S2):1-121. https://www. canada.ca/en/public-health/services/reports-publications/ canada-communicable-disease-report-ccdr/monthlyissue/2009-35/definitions-communicable-diseases-nationalsurveillance.html

8. Agence de la santé publique du Canada. Résumé : Estimations de l'incidence de la prévalence, et de la proportion non diagnostiquée au VIH au Canada, 2014. Agence de la santé publique du Canada, Ottawa, 2015. https://www.canada.ca/fr/sante-publique/services/ publications/maladies-et-affections/resume-estimationsincidence-prevalence-et-proportion-non-diagnostiquee-vihcanada-2014.html

9. Immigration, Refugees and Citizenship Canada, Immigration Medical Examination Instructions, HIV Screening. 1 November 2013.

10. National (Canadian) Collaborating Centre for Infectious Disease. Notifiable Disease Database. http://nddb.ca/ diseaseinfo/search/search_disease\#search_disease. [Consulté le 31 juil 2017]. 
11. Region SH, Virus HI. (HIV) - Testing, December 2016. http:// www.communityview.ca/pdfs/2016_shr_series5_hiv_testing. pdf. [Consulté le 31 juil 2017].

12. BC Centre for Disease Control. HIV in British Columbia: Annual Surveillance Report 2014, BC Centre for Disease Control, Vancouver, 2015.

13. Remis RS, Liu J. HIV/AIDS in Ontario: Preliminary Report, 2011, Toronto, August 2013. http://www.ohemu.utoronto.ca/ doc/PHERO2011_report_preliminary.pdf

14. Gouvernement du Canada Questions et réponses: Prévention des infections transmissibles sexuellement et par le sang chez les adultes âges. Agence de la santé publique du Canada, Ottawa, 2015. https://www.canada.ca/fr/santepublique/services/maladies-infectieuses/sante-sexuelle- infections-transmissibles-sexuellement/rapports-publications/ questions-reponses-adultes.html

15. Statistics Canada. Peuples autochtones - Faits saillants en tableaux, Recensement de 2016. http://www12.statcan. gc.ca/census-recensement/2016/dp-pd/hlt-fst/abo-aut/ Tableau.cfm? Lang $=$ Fra\& $T=101 \& S R=1 \& S=99 \& O=A \& R P P=25$ $\& P R=0 \& D 1=1 \& D 2=1 \& D 3=1 \& T A B I D=2$

16. Statistiques Canada. Recensement de la population de 2016, produit numéro 98-400-X2016190 au catalogue de Statistique Canada. hhttp://www12.statcan.gc.ca/censusrecensement/2016/dp-pd/dt-td/Rp-fra.cfm?TABID=2\&LANG $=F \& A=R \& A P A T H=3 \& D E T A I L=0 \& D I M=0 \& F L=A \& F R E E=0 \& G$ $C=01 \& G L=-1 \& G I D=1341679 \& G K=1 \& G R P=1 \& O=D \& P I D=11$ $0531 \& P R I D=10 \& P T Y P E=109445 \& S=0 \& S H O W A L L=0 \& S U B=$ $0 \&$ Temporal $=2017 \&$ THEME $=120 \& V I D=0 \& V N A M E E=\& V N A$ $\mathrm{MEF}=\& \mathrm{D} 1=0 \& \mathrm{D} 2=0 \& \mathrm{D} 3=0 \& \mathrm{D} 4=0 \& \mathrm{D} 5=0 \& \mathrm{D} 6=0$ 